\title{
Intrauterine device embedded into the bladder wall with stone formation: laparoscopic removal is a minimally invasive alternative to open surgery
}

\author{
Dong Gil Shin • Tae Nam Kim • Wan Lee
}

Received: 30 August 2011 / Accepted: 6 December 2011 /Published online: 22 December 2011

(C) The Author(s) 2011. This article is published with open access at Springerlink.com

\begin{abstract}
Spontaneous perforation of the uterus and intravesical intrauterine device (IUD) is very rare. The treatment options for an intravesical IUD are open surgery or cystoscopic removal. Open surgery has been used generally for the removal of IUDs with formation of big stones or partial penetration of the bladder wall. In this case, we removed an IUD (multiload $\mathrm{Cu} 375^{\circledR}$ ) with calculus that had sunk deeply into the bladder wall via laparoscopic partial cystectomy.
\end{abstract}

Keywords Intrauterine device · Laparoscopy · Urinary bladder calculi

\section{Introduction}

Perforation of the uterus and intravesical intrauterine device (IUD) is an infrequent complication. Once an IUD has penetrated the bladder, it usually becomes encrusted with calculi [1]. The treatment options for an intravesical IUD are varied. Open surgery is performed generally in cases of IUDs with partial penetration of the bladder wall, to remove the device and repair the defect [2]. However, this type of open surgery increases morbidity because of extensive surgical exploration. To reduce morbidity, we performed

D. G. Shin • T. N. Kim $(\bowtie)$

Department of Urology, Medical Research Institute,

Pusan National University Hospital,

1-10 Ami-Dong, Seo-gu,

Pusan 602-739, South Korea

e-mail: bigman1995@hanmail.net

W. Lee

Department of Urology, Cancer Center,

Dongnam Institute of Radiological \& Medical Science,

40 Jwadong-gil, Jangan-eup, Gijang-gun,

Pusan 619-953, South Korea laparoscopic removal of an intravesical IUD (multiload

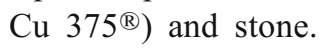

\section{Case report}

A 38-year-old woman was admitted to our clinic with a 2-year history of recurrent urinary tract infection, dysuria, dyspareunia, and chronic pelvic pain. She had had these symptoms six to seven times per year. Pelvic examination was normal, and the position of the uterus was anteverted and anteflexed in ultrasonography. Urinalysis revealed the presence of inflammation, but a urine culture remained sterile, with absence of crystals. There were no data to support a tendency of urolithiasis. The patient's medical history indicated that an IUD had been inserted in 2000 . Unexpectedly, she had become pregnant 10 months later, but aborted. The following year, she had become pregnant again and had given birth without complications. The IUD had not been found, and it was assumed that it had fallen out. When she presented at our clinic, it was the first time she was seen by a urologist. A plain abdominal radiograph showed the presence in the pelvis of a stone opacity with dimensions of $1.9 \times 1.4 \mathrm{~cm}$ around the IUD (Fig. 1). Pelvic computed tomography showed that a stone with dimensions of $1.6 \times 1.9 \mathrm{~cm}$ was located around the IUD in the left posterolateral part of the bladder. Cystoscopy revealed that the IUD was deeply embedded into the muscular wall of the bladder and that a bladder stone had formed around the IUD. We decided to perform laparoscopic removal of the IUD with stone formation because of the possibility of fistula, the possibility of laparoscopic repair of any bladder defect, and the decreased morbidity compared to open surgery. During the laparoscopic procedure, pervasive adhesions were seen between the posterior wall of the bladder, omentum, and 


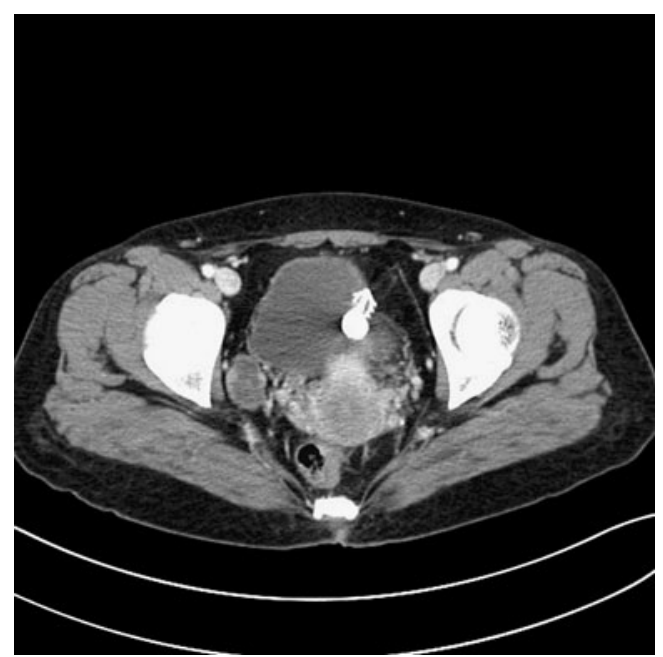

Fig. 1 Pelvic computed tomography shows $1.6 \times 1.9$-cm-sized stone around the IUD in the left posterolateral part of the bladder

uterine fundus. After the removal of the adhesions, we found that there was no fistula tract between the posterior wall of the bladder and the uterus. We incised the left posterolateral wall of the bladder, and the bladder stone around the IUD was exposed (Fig. 2). We removed the IUD and bladder stone via laparoscopic partial cystectomy. The bladder was closed using fine absorbable 4-0 sutures, in two layers. No leakage was observed after the bladder was filled with approximately $200 \mathrm{~mL}$ isotonic $\mathrm{NaCl}$. A drain was inserted, and the operation was concluded. Urethral Foley catheter was kept postoperatively for 6 days. Cystography performed on the seventh day showed that any contrast leakage in the bladder was not seen. No complications occurred in the postoperative period. The Foley catheter and drain were removed, and the patient was discharged on the eighth day.

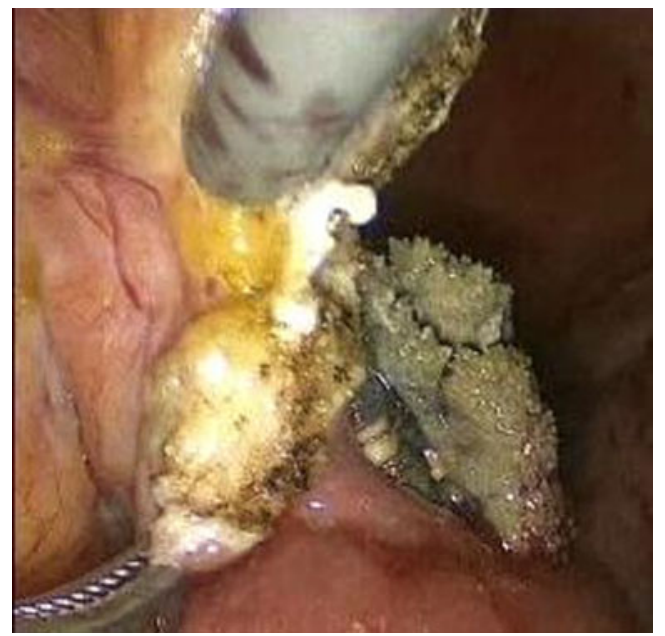

Fig. 2 The IUD with stone formation that had deeply sunk into the left posterolateral wall of the bladder

\section{Discussion}

The overall reported incidence of intrauterine device perforation is about 0.87 per 1,000 insertions [3]. Calculus formation develops over time in cases of IUDs that migrate into the bladder. They may be asymptomatic or yield abdominal or pelvic signs and symptoms, based on the severity of the problem and location of the IUD. The interval between insertion and symptoms varies from 6 months to 16 years [1]. In this case, the patient presented with dysuria and recurrent urinary tract infection that did not resolve completely after 7 years of IUD insertion, despite appropriate oral antibiotic therapy.

Any woman who has recurrent relapsing or persistent urinary tract infection warrants investigation including urinary tract imaging study. In the present case, the patient did not receive any imaging study of the urinary tract before she was admitted to our clinics.

Several mechanisms can explain the spontaneous migration of IUDs, including overlooked iatrogenic uterine perforation, spontaneous uterine contraction, involuntary bladder contraction, gut peristalsis, and peritoneal fluid movement. In our case, we could not ascertain the precise cause of the intrauterine IUD. Factors increasing the likelihood of uterine perforation include insertion of the device by inexperienced persons, inappropriate positioning of the IUD, susceptible uterine wall because of multiparity, and a recent abortion or pregnancy. Dysfunctional voiding symptoms with a history of an unretrieved IUD must be carefully researched for possible perforation of the uterus and intravesical IUD. The presence of bladder stones is unusual in women, which should raise the suspicion of the presence of a foreign body. When IUD strings are not observed in the vagina during examination and are not detected in the endometrial cavity using ultrasonography, a physician must consider IUD displacement.

Although there is controversy regarding the management of extrauterine IUDs in asymptomatic patients, there appears to be a consensus that all extrauterine copper-laden devices should be removed, as copper IUDs result in inflammatory reactions and adhesion. The World Health Organization (WHO) and the International Medical Advisory Panel Meetings of the International Planned Parenthood Federation recommend that an IUD displaced from the uterine cavity should be removed as soon as possible after establishment of the diagnosis, regardless of its type and location. Treatment options for IUDs that migrate into the bladder vary. Cystoscopic or suprapubic cystoscopic extraction of the device and stones can be useful for IUDs that are located completely inside the bladder, or for IUDs that exhibit formation of small calculi [4]. Open surgery has been used generally for the removal of IUDs with formation of big stones or with partial penetration of the bladder wall $[2,5]$. 
However, open surgery entails increased patient morbidity. Therefore, treatment options for displaced IUDs must be chosen carefully because of the increase in morbidity entailed by open surgery and because of patient preferences for the least invasive procedure. In the present case, we removed the IUD via laparoscopic simple excision of the bladder wall around the IUD after cleavage between the bladder and the uterus, as the IUD had sunk deeply into the muscular layer, with the possibility of formation of a vesicouterine fistula. In conclusion, chronic pelvic pain and irritative voiding symptoms with a history of an unretrieved IUD must be carefully researched for possible perforation of the uterus and intravesical IUD. An IUD that migrates into the bladder must be removed because of associated complications. Laparoscopy can be a less invasive option than an open surgical procedure.

\section{Conflicts of interest None.}

Open Access This article is distributed under the terms of the Creative Commons Attribution Noncommercial License which permits any noncommercial use, distribution, and reproduction in any medium, provided the original author(s) and source are credited.

\section{References}

1. Dietrick DD, Issa MM, Kabalin JN, Bassett JB (1992) Intravesical migration of intrauterine device. J Urol 147:132-134

2. El-Hefnawy AS, El-Nahas AR, Osman Y, Bazeed MA (2008) Urinary complications of migrated intrauterine contraceptive device. Int Urogynecol J Pelvic Floor Dysfunct 19:241-245

3. Markovitch O, Klein Z, Gidoni Y, Holzinger M, Beyth Y (2002) Extrauterine mislocated IUD: is surgical removal mandatory? Contraception 66:105-108

4. Atakan H, Kaplan M, Erturk E (2002) Intravesical migration of intrauterine device resulting in stone formation. Urology 60:911

5. Maskey CP, Rahman M, Sigdar TK, Johnsen R (1997) Vesical calculus around an intra-uterine contraceptive device. Br J Urol 79:654-655 